\title{
Erratum: The automation of next-to-leading order electroweak calculations
}

\author{
R. Frederix, ${ }^{a, 1}$ S. Frixione, ${ }^{b}$ V. Hirschi, ${ }^{c, 2}$ D. Pagani, ${ }^{a, 3}$ H.-S. Shao ${ }^{d}$ and M. Zaro ${ }^{e, 4}$ \\ ${ }^{a}$ Physik Department T31, Technische Universität München, \\ James-Franck-Str. 1, D-85748 Garching, Germany \\ ${ }^{b}$ INFN, Sezione di Genova, \\ Via Dodecaneso 33, I-16146, Genoa, Italy \\ ${ }^{c}$ Institute for Theoretical Physics, ETH Zürich, \\ Wolfgang-Pauli-strasse 27, 8093 Zürich, Switzerland \\ ${ }^{d}$ Laboratoire de Physique Théorique et Hautes Energies (LPTHE), \\ UMR 7589, Sorbonne Université et CNRS, \\ 4 place Jussieu, 75252 Paris Cedex 05, France \\ ${ }^{e}$ Nikhef, \\ Science Park 105, NL-1098 XG Amsterdam, The Netherlands \\ E-mail: rikkert.frederix@tum.de, Stefano.Frixione@cern.ch, \\ hirschva@itp.phys.ethz.ch, davide.pagani@tum.de, \\ huasheng.shao@lpthe.jussieu.fr,m.zaro@nikhef.nl
}

ERRATUM TO: JHEP07(2018)185

ARXiv EPrint: 1804.10017

\footnotetext{
${ }^{1}$ Now at: Theoretical Particle Physics, Department of Astronomy and Theoretical Physics, Lund University, Sölvegatan 14A, SE-223 62 Lund, Sweden; e-mail: rikkert.frederix@thep.lu.se.

${ }^{2}$ Now at: Theoretical Physics Department, CERN, CH-1211 Geneva 23, Switzerland; e-mail: valentin.hirschi@cern.ch.

${ }^{3}$ Now at: INFN, Sezione di Bologna, Via Irnerio 46, 40126 Bologna, Italy; and DESY, Theory Group, Notkestrasse 85, 22607 Hamburg, Germany; e-mail: davide.pagani@bo.infn.it.

${ }^{4}$ Now at: TIFLab, Università degli Studi di Milano \& INFN, Sezione di Milano, Via Celoria 16, 20133 Milano, Italy; e-mail: marco.zaro@mi.infn.it.
} 
In this erratum we amend the values of some of the input parameters quoted in section 6 of ref. [1], which differ from those used to obtain the cross-section results presented therein.

In particular, the values of the widths $\Gamma_{Z}^{\mathrm{BW}}, \Gamma_{W}^{\mathrm{BW}}$ reported in eqs. (6.4) and (6.5) must read as follows (in accordance with ref. [2], cited as ref. [105] in the paper):

$$
\begin{array}{ll}
M_{Z}^{\mathrm{BW}}=91.1876 \mathrm{GeV}, & \Gamma_{Z}^{\mathrm{BW}}=2.4952 \mathrm{GeV}, \\
M_{W}^{\mathrm{BW}}=80.385 \mathrm{GeV}, & \Gamma_{W}^{\mathrm{BW}}=2.0850 \mathrm{GeV} .
\end{array}
$$

We are indebted to Pia Bredt for having reported this discrepancy.

Open Access. This article is distributed under the terms of the Creative Commons Attribution License (CC-BY 4.0), which permits any use, distribution and reproduction in any medium, provided the original author(s) and source are credited.

\section{References}

[1] R. Frederix, S. Frixione, V. Hirschi, D. Pagani, H.S. Shao and M. Zaro, The automation of next-to-leading order electroweak calculations, JHEP 07 (2018) 185 [arXiv:1804.10017] [INSPIRE].

[2] Particle Data Group collaboration, Review of Particle Physics, Chin. Phys. C 40 (2016) 100001 [inSPIRE]. 